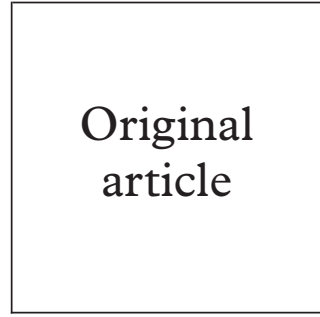

\author{
Centers for Disease \\ Control and \\ Prevention, Public \\ Health Service, US \\ Department of Health \\ and Human Services, \\ Atlanta, GA, USA \\ $S$ D Vernon \\ E R Unger \\ S Z Wiktor \\ D L Miller \\ A E Greenberg \\ W C Reeves
}

National Cancer

Institute, Cancer

Prevention Fellowship

Office, Rockville, MD,

USA

M A Piper

Projet RETRO-CI,

CHU Treichville,

Abidjan, Côte d'Ivoire

$\mathrm{S} T$ Severin

S Z Wiktor

P D Ghys

A E Greenberg

Institute of Tropical

Medicine, Antwerp,

Belgium

P D Ghys

Emory School of Medicine, Emory

University, Atlanta,

GA, USA

I R Horowitz

Correspondence to:

Suzanne D Vernon, PhD,

Centers for Disease Control

and Prevention, 1600 Clifton

Road, MSG18, Atlanta, GA

30333, USA.

Accepted for publication

24 May 1999

\title{
HIV and human papillomavirus as independent risk factors for cervical neoplasia in women with high or low numbers of sex partners
}

Suzanne D Vernon, Elizabeth R Unger, Margaret A Piper, Sibailly T Severin, Stefan Z Wiktor, Peter D Ghys, Donna L Miller, Ira R Horowitz, Alan E Greenberg, William C Reeves

Objective: To explore whether HIV types 1 and 2 and CD 4 cell count affect cervical neoplasia independent of human papillomavirus (HPV) in women with high or low numbers of sexual partners residing in Abidjan, Côte d'Ivoire.

Methods: The study population and methods are described in the companion paper. Additional methods include a Papanicolaou smear for cytological diagnosis and statistical analysis.

Results: In maternal women, both HIV-1 and high risk HPV were significant independent risk factors for squamous intraepithelial lesions (SIL) (adjusted odds ratio (OR) 11.0 (95\% CI 1.1112 ) and 5.4 (1.5-18.8), respectively). Only high levels of HPV DNA in the lavage were associated with SIL (OR 13.2 (3.6-47.8)) in the maternal group. In female sex workers, high risk HPV was significantly associated with SIL (OR 23.7 (4.4-126)); HIV seropositivity was not. Any positive level (high or low amounts) of HPV DNA was significantly associated with SIL in sex workers (ORs 15.9 (3.3-76) and 12.7 (3.6-44), respectively). There was no association of SIL with CD4 cell counts $\leqslant 500 \times 10^{6} / 1$ in HIV seropositive women from either group.

Conclusion: HPV or HIV-1 infection independently affect cervical neoplasia in women with low numbers of sex partners.

(Sex Transm Inf 1999;75:258-260)

Keywords: HIV; human papillomavirus; cervical neoplasia

\section{Introduction}

Increased rates of preinvasive cervical neoplasia have been noted repeatedly in women infected with HIV compared with uninfected controls. ${ }^{12}$ Most studies linking HIV infection to human papillomavirus (HPV) and cervical neoplasia have focused on populations of women with high risk sexual behaviours. Since sexual practices are strongly associated with both cervical disease and HIV transmission, it is difficult to determine if the association is due to shared risk factors or to a biological effect. ${ }^{3}$ In our companion paper, we observed different associations between HIV and HPV that depended on numbers of sex partners and CD 4 cell count. ${ }^{4}$ Here we examine whether HIV and HPV can be independent risk factors for cervical neoplasia.

\section{Methods}

STUDY POPULATIONS AND METHODS

The study populations and methods were described in the companion article. ${ }^{4}$

\section{CERVICAL CYTOPATHOLOGY}

Papanicolaou smear results were reported by using the Bethesda system. ${ }^{5}$ For data analysis, low grade and high grade squamous intraepithelial lesions (SIL) were grouped as SIL. Inflammatory and reactive changes were classified with normal smears. Unsatisfactory samples were excluded from the analysis.

\section{DATA ANALYSIS}

For evaluation of the association between HIV and SIL, women were classified into groups according to their serostatus (negative, HIV-1, HIV-2, or HIV-D). The odds of SIL were estimated for each HIV exposure group relative to HIV negative women. A sample was considered HPV positive when the relative light unit (RLU) ratio (RLU of sample/mean RLU of three positive controls (PC)) was $\geqslant 1 .{ }^{6}$ To determine if SIL was associated with HPV viral load, we grouped samples with positive high risk results as either $1-20 \mathrm{RLU} / \mathrm{PC}$ or $>20$ RLU/PC.

\section{Results}

ABNORMAL CERVICAL CYTOLOGY RATES

In the maternal group, $10 / 56(18 \%, \mathrm{p}<0.001)$ HIV-1, 4/55 (7\%, $\mathrm{p}=0.02) \mathrm{HIV}-2,3 / 21(14 \%$, $\mathrm{p}<0.001)$ HIV-D, and $1 / 119(0.08 \%$, reference) seronegative women had SIL. By contrast, SIL rates among sex workers did not differ significantly. Among sex workers, 11/109 (10\%, $\mathrm{p}=0.14)$ HIV-1, 5/63 HIV-D (8\%, $\mathrm{p}=0.4)$, and $3 / 68(4 \%$, reference) seronegative women had SIL. Abnormal Pap smears interpreted as atypical squamous cells of undetermined significance (ASCUS) were not significantly different between HIV seropositive and HIV seronegative women in either group (data not shown).

ASSOCIATION OF HIV SEROSTATUS AND HPV WITH SIL AND ASCUS

The association of HIV serostatus or HPV with SIL is shown in table 1 . In the maternal women, both HIV-1 and high risk HPV DNA detection were significantly and independently 
Table 1 Association of SIL ${ }^{\star}$ with HIV types and HPV in maternal women and female sex workers

\begin{tabular}{|c|c|c|c|c|}
\hline & \multicolumn{2}{|c|}{ Maternal women } & \multicolumn{2}{|c|}{ Female sex workers } \\
\hline & Freq (\%) & ORt $(95 \% C I)$ & Freq (\%) & OR+ $(95 \% C I)$ \\
\hline HIV-1 & $10 / 48(21)$ & $11.0(1.1-112.0)$ & $11 / 91$ & $0.6(0.1-5.0)$ \\
\hline HIV-2 & $4 / 51(8)$ & $3.9(0.4-40.0)$ & 0 & - \\
\hline HIV-D & $3 / 17(18)$ & $7.1(0.6-9.4)$ & $5 / 57(9)$ & $0.4(0.1-1.4)$ \\
\hline HIV-neg & $1 / 107(1)$ & Reference & $3 / 60(5)$ & Reference \\
\hline High risk HPV & $14 / 62(23)$ & $5.4(1.5-18.8)$ & $16 / 64(25)$ & $23.7(4.4-126.0)$ \\
\hline Low risk HPV & $0 / 14(0)$ & - & $1 / 15(7)$ & $4.4(0.3-57.0)$ \\
\hline Negative HPV & $4 / 146(3)$ & Reference & $3 / 155(2)$ & Reference \\
\hline
\end{tabular}

^SIL versus normal (ASCUS excluded).

HOR were adjusted for HIV type, HPV, age, number of lifetime sex partners (maternal group), smoking (sex workers), and education.

- No SIL present for reference or variable value.

Table 2 Association of SIL with high risk HPV DNA level

\begin{tabular}{|c|c|c|c|c|}
\hline \multirow{2}{*}{$\begin{array}{l}H P V D N A \\
\text { (RLU ratio) }\end{array}$} & \multicolumn{2}{|l|}{ Maternal women } & \multicolumn{2}{|l|}{ Female sex workers } \\
\hline & $O R^{\star}(95 \% C I)$ & $p$ Value & $O R^{\star}(95 \% C I)$ & $p$ Value \\
\hline $1-20$ & $1.4(0.2-8.3)$ & 0.7 & $12.7(3.6-44.0)$ & 0.0001 \\
\hline$>20$ & $13.2(3.6-47.8)$ & 0.0001 & $15.9(3.3-76.0)$ & 0.0005 \\
\hline
\end{tabular}

${ }^{\star}$ Controlling for HIV-1, HIV-2, HIV-D.

associated with SIL. In female sex workers, only high risk HPV detection was significantly associated with SIL. In the maternal women, ASCUS was not significantly associated with any of the variables examined (data not shown). In the female sex workers, high risk HPV detection was significantly associated with ASCUS (OR 2.9 (1.1-7.3)).

LOW CD4 COUNTS AND SIL

To determine if low CD4 counts were associated with SIL, we did a stratified univariate analysis of HIV-1 seropositive women. Among HIV-1 seropositive maternal women, there was no association of SIL with CD 4 counts $\leqslant 500$ $\times 10^{6} / 1$ (OR $2.5(0.4-16)$ ). Similarly, no association of SIL with CD 4 cell counts $\leqslant 500 \times 10^{6} / 1$ was detected among the HIV-1 seropositive sex workers (OR $2.5(0.5-18)$ ).

HIGH RISK HPV DNA LEVELS

We determined if the level of high risk HPV DNA had an effect on SIL (table 2). Only high levels of HPV DNA were found to be significantly associated with SIL in maternal women (OR 13.2 (3.6-47.8)). Among sex workers, both low $(1-20)$ and high $(>20)$ levels of HPV DNA were significantly associated with SIL (ORs 12.7 (3.6-44), 15.9 (3.3-76) respectively).

\section{Discussion}

We examined whether HIV serostatus and HPV could be independent risk factors for cervical neoplasia in women with high or low numbers of sexual partners. We found that HIV-1 could be an independent risk factor for SIL in the maternal women but not in the sex workers. Why HIV-1 was a risk factor in the maternal women but not in the sex workers is not clear. Smith et $a l^{7}$ studied a high risk population and found that in the absence of immunosuppression there was no difference in the rate of SIL or HPV between HIV seropositive and seronegative women. Only one other study has examined the relation between HIV infection and cervical disease in a low risk population. ${ }^{8}$ These investigators observed a significant relation between HIV infection and cervical disease but did not measure HPV or any other risk factor associated with cervical disease. HIV serostatus in the maternal women could be a surrogate marker for a factor(s) of cervical disease that we did not measure.

We did not find HIV-2 seropositivity in maternal women to be associated with SIL in multivariate analysis. One other group has studied HIV-2 and cervical neoplasia in high risk African women and found that SIL was associated with HIV-2 infection. ${ }^{9}{ }^{10}$ In their analysis, ORs were adjusted for number of sex partners per week and study site, but not for HPV infection. Even though HIV-1 and HIV-2 are both sexually transmitted, the epidemiology of HIV-2 is different. Slower heterosexual spread, lower infectivity and lower HIV-2 viral load compared with HIV- ${ }^{10}$ may be related to the lack of a measurable effect of HIV-2 on cervical neoplasia in our study.

The strong association between SIL and high risk HPV DNA in both groups was expected. It was surprising, however, to find that SIL was associated with different amounts HPV DNA detected in these two groups. The amount of HPV DNA may vary depending on a number of factors specific to the population such as sexual behaviour, ${ }^{4}$ or the specific HPV type(s) that predominate in a population. ${ }^{11}$ This indicates the difficulty in using HPV quantitation for distinguishing clinically significant $\mathrm{HPV}$ infections.

Most studies attribute the increased rates of HPV associated cervical disease in HIV infected women to immunosuppression. In the results reported here, immunosuppression as measured by CD 4 cell counts of $\leqslant 500 \times 10^{6} / 1$ was not associated with SIL in either group. Admittedly, very few women had very low CD4 cell counts, and this may be one explanation for the relatively low rates of SIL observed in both groups. However, if we analysed SIL among only the HIV-1 seropositive women with CD4 $\leqslant 500 \times 10^{6} / 1$, low CD 4 counts did not account for all the SIL detected in HIV-1 seropositive women from either group. It may be that in the absence of significant immunosuppression, HIV alters pathogenesis of HPV associated cervical disease. ${ }^{12}$

The observation that HIV-1 seropositivity is an independent risk factor for SIL in women with low numbers of sexual partners needs to be confirmed. Our study is limited by differences inherent in these two groups and factors we did not measure, such as diet and nutritional status. Despite these limitations, it is intriguing to once again observe that HIV-1 may directly or indirectly alter pathogenesis of cervical disease.

The authors gratefully acknowledge the staff at Projet RETRO $\mathrm{CI}$ and Clinique de Confiance for their support in the clinics, Co oulibaly Kady, and Dr Marie Laga fo supporting this study effort. We also would like to thank D Rosane Nisenbaum for analytical assistance.

All study participants were volunteers who gave informed consent. Complete study protocols were approved by the 
Ivorian Ministry of Health and Centers for Disease Control and tion guidelines of the US Department of Health and Human Services were followed in the conduct of this study.

Contributors: SD Vernon, ER Unger, and WC Reeves conceived the study and together with SZ Wiktor and AE Greenberg, implemented the study design and carried it out. ST Severin and PD Ghys ran the clinics and cared for the women. IR Horowitz provided gynaecological training. ER Unger participated in pathology assessments. L Miller provided laboratory expertise and performed HPV tests. MA Piper performed statistical analysis. SD Vernon and ER Unger co-wrote the statistica

1 Laga M, Icenogle JP, Marsella R, et al. Genital papillomavirus infection and cervical dysplasia - opportunistic complications of HIV infection. Int $\mathcal{f}$ Cancer 1992;50:45-8.

2 Wright TC, Ellerbrock TV, Chiasson MA, et al. Cervical intraepithelial neoplasia in women infected with human immunodeficiency virus: prevalence, risk factors, an validity of Papanicolaou smears. Obstet Gynecol 1994;84: 591-7

3 Kjaer SK, De Villiers EM, Haugaard BJ, et al. Human papillomavirus, herpes simplex virus and cervical cancer incidence in Greenland and Denmark. A population-based cross-sectional study. Int 7 Cancer 1988;41:518-24.
4 Piper MA, Severin ST, Wiktor SZ, et al. Association of human papillomavirus with HIV and CD4 cell count in women with high or low numbers of sex partners. Sex Transm Inf 1999;75:253-7.

5 National Cancer Institute Workshop. The 1988 Bethesda system for reporting cervical/vaginal cytologic diagnoses. ҰAMA 1989;262:931-4

6 Lörincz A. Diagnosis of human papillomavirus infection by the new generation of molecular DNA assays. Clin Immunol Newsletter 1992;12:123-8.

7 Smith JR, Kitchen VS, Botcherby M, et al. Is HIV infection associated with an increase in the prevalence of cervical neoplasia? Br f Obstet Gynecol 1993; 100:149-53.

8 Magrwa BN, Hunter DJ, Mbugua S, et al. The relationship between HIV infection and cervical intraepithelial neoplabetween $\mathrm{HI}$ infection and cervical intraepithelial neoplasia among women attending two family
Nairobi, Kenya. AIDS 1993;7:733-8.

9 Langley CL, Benga-De E, Critchlow CW, et al. HIV-1, Langley CL, Benga-De E, Critchlow CW, et al. HIV-1,
HIV-2, human papillomavirus infection and cervical neoplasia in high risk African women. AIDS 1996;10:41317

10 Kanki PJ, Travers KU, Mboup S, et al. Slower heterosexual spread of HIV-2 than HIV-1. Lancet 1994;343:943-6.

1 Acs J, Hildesheim A, Reeves WC, et al. Regional distribution of human papillomavirus DNA and other risk factors for invasive cervical cancer in Panama. Cancer Res 1989;49. 5725-9.

12 Vernon SD, Hart CE, Reeves WC, et al. The HIV-1 tat protein enhances E2-dependent human papillomavirus 16 transcription. Virus Res 1993;27:133-45. 